\title{
High copy number variations, particular transcription factors, and low immunity contribute to the stemness of prostate cancer cells.
}

\section{Zao Dai}

College of Life Sciences, Nanjing Normal University, Nanjing, Jiangsu, China

Ping Liu ( $\square$ liuping0805@njnu.edu.cn)

College of Life Sciences, Nanjing Normal University, Nanjing, Jiangsu, China https://orcid.org/00000001-5366-4618

\section{Research}

Keywords: stemness of prostate cancer, WGCNA, ATAC-seq, CNV, immune infiltration

Posted Date: February 2nd, 2021

DOI: https://doi.org/10.21203/rs.3.rs-100256/v2

License: (c) (i) This work is licensed under a Creative Commons Attribution 4.0 International License.

Read Full License 


\section{Abstract}

Background: Tumor metastasis is the main cause of death of cancer patients, and the existence of cancer stem cells is the basis of tumor metastasis. However, the systematic analysis of the stemness of prostate cancer cells is still not abundant. This article explores the effective factors that lead to the stemness of prostate cancer cells with multi-omics data mining.

Methods: Gene expression modules that were strongly related to the stemness of prostate cancer cells were obtained with WGCNA and stemness scores, based on the prostate cancer transcriptome data in TCGA. Calculated the copy number variation of stemness genes of prostate cancer and evaluated the difference of transcription factors in prostate cancer and normal tissues, based on CNV (copy number variation) data and ATAC-seq data. The protein interaction network of stemness genes of prostate cancer was constructed with the STRING database. At the same time, the correlation between stemness genes of prostate cancer and immune cells was analyzed.

Results: Prostate cancer with high Gleason grade was more stemness. The gene set highly related to prostate cancer stemness had higher $\mathrm{CNV}$ in prostate cancer samples than normal samples. The transcription factors of stemness genes had similar expressions but had different contributions in normal and prostate cancer tissues. And particular transcription factors enhanced the stemness of prostate cancer, such as PUM1, CLOCK, SP1, TCF12, and so on. The lower tumor immune microenvironment was conducive to the stemness of prostate cancer, and CD8+ T cells and M1 macrophages might play an important role than other immune cells in the tumor microenvironment. We also found that EZH2 played a central role in stemness genes and was negatively correlated with resting mast cells and positively correlated with activated memory CD4+ T cells.

Conclusions: This study found that high copy number variation, specific transcription factors, and low immune microenvironment contributed to the stemness of prostate cancer cells through systematic multiomics analysis. These findings would provide new clues and directions for future research of prostate cancer stem cells.

\section{Background}

Cancer stem cells (CSCs) are a few stemness-like cells with the ability of self-renewal and differentiation into cancer cells [1]. They play an important role in the occurrence and development of tumors, especially closely related to tumor metastasis [2-4]. In prostate cancer, the stemness of cancer cells (including prostate cancer stem cells, PCSC) is closely related to prostate cancer's metastasis [5]. In the process of prostate cancer metastasis, PCSCs initiates EMT (epithelial-mesenchymal transition) to form fibroblastlike cells and then enter the blood. With the circulation system, prostate cancer cells migrate to other tissues (such as bone tissue and lymph tissue) and grow into tumor tissue in other tissues, which leads to tumor metastasis (cancer cell spreading). 
As we are known, many factors are related to the stemness of CSC cells, not only including intracellular factors (such as stemness-related genes) but also including the microenvironment of cancer tissues (such as immune cells in the tumor microenvironment) [3, 6, 7]. In prostate cancer, it has been reported that the immune cells (especially CD8+ T cells and macrophages) in the microenvironment of prostate cancer are closely related to the metastasis of PCa cells $[8,9]$. The number of immune cells around the early PCa tissue will decrease with the growth of the cancer tissue, which results in the immunity in the microenvironment of PCa also decrease [10]. With the development of PCa to the later stage (Gleason score to 6-10), some studies report that some immune cells (such as related T cells) in the microenvironment can reverse to promote or enhance the growth of PCa cells and help cancer cells metastasize [11].

Although increasing publications have reported the relationship between stemness and metastasis in PCa cells, few studies on the stemness regulation of PCa cells [12,13]. Lots of factors affecting the stemness of PCa cells remain unclear and need to investigate.

The bioinformatics method based on the TCGA database has been increasingly used to analyze the molecular basis of prostate cancer development and clinical patient prognosis [14-16]. By using appropriate analytical software and methods to explore a variety of large-size data of clinical specimen from the TCGA database (including transcriptome sequencing data, gene sequencing data, ATAC-seq data, etc.), the molecular basis of prostate tumorigenesis, development of PCa, and the prognosis of patients might be figured out $[17,18]$. Therefore, the bioinformatics analysis of TCGA data should help provide clues and direction for basic experimental research and clinical cancer treatment in PCa.

This study obtained the most important stemness-related modules and genes in prostate cancer cells from transcriptome data and OCLR scores [2] (for scoring the stemness of tumors).

Further analysis showed that stemness-related genes had higher CNV in prostate cancer than prostate normal samples. And transcription factors of stemness genes enhanced the stemness of prostate cancer cells. The analysis results showed that the stemness of prostate cancer cells was negatively correlated with the immune response, and low immune scores were beneficial to the prostate cancer stemness. We also found that EZH2 played a central role in these stemness genes. Our multi-omics data analysis results provided the theoretical basis and directions for subsequent research on prostate cancer stemness.

\section{Methods}

\section{Analysis of Clinical data}

Obtained the clinical information of prostate cancer in the TCGA database. According to the Gleason score, we divided prostate cancer samples into five grades, namely Gleason grade 1 (Gleason score 6), Gleason grade 2 (Gleason score (3+4)), Gleason grade 3 (Gleason score(4+3)), Gleason grade 4 (Gleason score 8), Gleason grade 5 (Gleason score 9 or 10). 


\section{Analysis of transcriptome (RNA-seq) data}

Based on OCLR stemness scores and Gleason grade of TCGA prostate cancer clinical data, correlation analysis between PCa cell stemness and Gleason grade was carried out. By combing the stemness score, WGCNA [19] analysis was performed on the transcriptome data of prostate cancer in TCGA. Differential expression analyses of genes in the WGCNA results most relevant to PCa cell stemness were performed and presented in a heatmap. Further, normalized expression of stemness genes was obtained with the between-array normalization in the limma [20] package from the transcriptome data of 33 samples (GSE104786) in the GEO database, and a heatmap was drawn. Also, the transcriptome data of the SRA database (Normal samples(SRR7651698, SRR7651699, SRR7651700), PCa cell lines (SRR7651715, SRR7651716, SRR7651717, SRR7651718), and other small cell prostate cancer (SRR7651719, SRR7651720)) were aligned with HISAT2 [20] and were quantified with HTSeq [21]. Finally, the normalized expression data was obtained with the TPM (Transcript per million) normalization method.

\section{Analysis of gene CNV data}

The genes most related to stemness in WGCNA results were screened, and their locations in genome locations were obtained by the local Perl script method. Combined with the CNV data of TCGA, the local Perl script was used to screen the segments containing the locations of important stemness genes. The prostate cancer samples were first classified by Gleason grade and then the CNV of essential stemness genes in each classified prostate cancer samples were calculated by using GISTIC2.0 [22].

\section{Analysis of ATAC-seq data}

From TCGA and SRA database, we got the ATAC-seq data (including normal prostate samples (SRR7651660, SRR7651661, SRR7651662) and prostate cancer samples (SRR7651675, SRR7651676, and TCGA ATAC-seq alignment results) data). Then $2 \mathrm{~kb}$ data of upstream and downstream of the important stemness genes were obtained using Bowtie2 software [23]. According to the results, the TSS signal intensity of stemness genes was drowned by using deeptools [24]. After analyzing the above alignment data using MACS [25] and HOMER [26], information about transcription regulators of the stemness genes was obtained; and the importance of each transcriptional factor was further obtained from TCGA transcriptome data by PCA analysis with R language. For example, the upstream transcriptional factor of EZH2 was drawn with Sushi [27] package in $\mathrm{R}$ language.

\section{Analysis of tumor microenvironment and immune infiltration}

The tumor microenvironment score and tumor immune infiltration score were calculated with ESTIMATE [28] and CIBERSORT [29]. Then, the correlation between stemness score and the immune score of PCa cells was calculated with R by combining with Gleason grade of prostate cancer. Based on CIBERSORT analysis results, the distribution map of immune cell components of different Gleason 
grades in prostate cancer was drawn with $\mathrm{R}$. The correlation network among immune cells was also drawn with $\mathrm{R}$ according to the correlation and significance of different immune cells.

\section{Protein interaction network and the correlation between stemness genes and immune infiltration}

After getting and analyzing PPI (Protein-Protein Interaction) data from the STRING (functional protein association networks) database, the PPI data of essential stemness genes were obtained. The interaction network of proteins of essential stemness genes was drawn with Cytoscape [30]. GO and KEGG enrichment analysis of important stemness genes were also carried out with ClusterProfiler [31]. And the results were combined with transcription data of essential stemness genes and the consequences of immune infiltration to analyze and calculated the correlation between the critical stemness genes and immune cells with $\mathrm{R}$.

\section{Results}

\section{Stemness-related key genes are highly expressed in high Gleason grade prostate cancer samples}

The results showed that the stemness of PCa cells of Gleason grade 3-5 was significantly higher than that of Gleason grade 1-2 (Fig. 1a), based on the OCLR's results and transcriptome data of PCa clinical specimen in TCGA. Besides, by using WGCNA (Weighted Gene Co-expression Network Analysis) to analyze the PCa transcriptome data for cancer cell stemness-related gene transcripts, we got 30 transcript modules related to PCa cell stemness (Fig. 1b), and many samples were clustered into different modules (Supplementary Fig. 1a). About $88.2 \%$ of the 2158 known cell stemness-related genes [32] were found in the results of our WGCNA analysis (Supplementary Materials S1; Supplementary Fig. 1c). Among these modules, we found that the MEmagenta module was most related to the stemness of prostate cancer cells (Fig. 1b), and the genes in the MEmagenta module were also positively correlated with stemness (Supplementary Fig. 1b). Therefore, the genes in the MEmagenta module (Supplementary Table S1) might play a vital role in the stemness of prostate cancer.

From the analysis results, we found that the expression of genes in the MEmagenta module in PCa cancer specimen was generally higher than that in the normal prostate specimen. Expression of these stemness genes in PCa increased with Gleason grade, and the highest expression was found at Gleason grade 5 (Fig. 1C). Further, we analyzed the data from 33 samples of the GEO database and found that most of the stemness genes expression in small cell prostate cancer (SCPC) were higher than those in prostate adenocarcinoma (PRAD) (Supplementary Fig. 1d). Subsequently, the transcriptome data of different prostate (normal or PRAD) cell lines and SCPC cell lines were analyzed, and it's found that the expression of stemness gene in PCa cells (PRAD cell lines) was higher than that in normal cells. Simultaneously, the expression of stemness genes in SCPC cell lines was slightly higher than in PRAD cell lines (Supplementary Fig. 1e).

In the genes of the MEmagenta module (Supplementary Table S1), we found that the known genes closely related to cancer stemness in non-prostate cancer, such as BRCA1 [33], EZH2 [33], FOXM1 [34], 
CDC20 [35], and CDCA8 [36] were all clustered in this module, indicating these genes were also closely related to the stemness of prostate cancer cells. From supplementary table $\mathrm{S} 1$, we also found that EZH2 was the most significant gene in the correlation with the stemness of PCa cells.

\section{The CNV of stemness-related genes elevated in prostate cancer}

The segments containing all MEmagenta module genes were obtained from TCGA and then analyzed using the GISTIC (Genome Identification of Significant Targets in Cancer) method. Analysis results showed that most of the genes in the MEmagenta module were found to have high variations, which were distinctly higher than those in normal prostate tissue, in prostate cancer, indicating that the stemness of PCa cells was influenced by the CNVs (Copy Number Variations) of these genes (Fig. 2a and Supplementary Fig. 2a). In two types of CNV (amplification and deletion, Supplementary materials S2S3), the change of deletion CNVs was more than amplification CNVs of the genes in the MEmagenta module of PCa samples. Both amplification and deletion CNVs in PCa samples were much higher than those in prostate normal samples (Fig. $2 \mathrm{~b}$ and $2 \mathrm{c}$, and Supplementary Fig. $2 \mathrm{~b}$ and Supplementary Fig. 2c). Besides, by combining analysis with Gleason grade of PCa, GISTIC results showed that CNVs of genes in the MEmagenta module also increased with the Gleason grade (Fig. 2d), suggesting that CNVs of genes in the MEmagenta module were related to the malignancy of PCa.

In details of CNVs of some genes in the MEmagenta module, it was found that the CNVs (amplification) and expression of SKA3 and RUVBL1, which promoted tumor metastasis and played a role in the development of stem cells [37-40], were all increased with the Gleason grade (Supplementary Fig. $3 a$ and $3 b$ ); and the CNVs (deletion) and expression of MCM6 and CENPH, which enhanced cancer cell proliferation, stemness, and metastasis and promoted cancer development [41-45], were also increased with the increase of Gleason grade (Supplementary Fig. 3c and Supplementary Fig. 3d).

\section{Transcription factors of stemness-related genes enhanced the stemness of PCa cells}

ATAC-seq data of the MEmagenta module genes in PCa samples obtained from TCGA and the sequence data of the $2 \mathrm{~kb}$ range of the transcription start site (TSS) were analyzed and displayed. Results showed that both normal and tumor prostate samples had the binding signals of transcription factors. The binding signals of all PCa samples (including the different Gleason grades of PCa and small cell $\mathrm{PCa}$ ) were weaker than that of normal prostate samples (Fig. 3a), and the binding signals of transcription regulators in small cell PCa was the weakest ones (Fig. 3a). These results indicated that the transcriptional regulation manner and the types of transcription regulators of the MEmagenta module genes in PCa might be different from those in normal prostate tissues.

According to the ATAC-seq data analysis results, it was found that most transcription factors of stemness genes in prostate samples were the same (Supplementary Fig. 4b). After filtering the 238 common transcription factors according to the genome information and transcriptome data, the expression profile of 163 transcription factors was finally obtained. After clustering the transcriptome data of the common 163 transcription factors, 3 clusters were obtained according to the expression of 
163 common transcription factors (Supplementary Fig. 4a). The first cluster has 86 transcription factors (such as FOXA1, HOXB13, ERG1, and so on), the second cluster has 44 transcription factors (such as SOX15, FOXM1, SOX2, and so on), and the third cluster has 33 transcription factors (such as NANOG, SOX3, FOXA2 and so on) (Supplementary Fig. 4a). In the top ten results of principal component analysis (PCA) of 163 common transcription factors, it was found that stemness-related PUM1 [46, 47], CLOCK [48], SP1, and TCF12 played a major positive regulation role on PCa cell stemness (Fig. 3b and Supplementary Fig. 4c). In comparison, IRF3 [49] negatively correlated with other 9 transcription factors and played a negative regulatory role on PCa cell stemness (Fig. 3b). As we know, IRF3 (interferon regulatory factor 3) signaling played an essential role in TLR3-mediated apoptosis in LNCaP cells through the activation of the intrinsic and extrinsic apoptotic pathways [49], suggesting that the immune system might play a role in suppressing the stemness of PCa cells. Furthermore, our analysis results showed that AR, FOXA1, NFYA, CTCF, and FOXO3 might enhance the stemness of PCa cells, where FOXF1 might be negatively correlated with these transcription factors (Fig. 3c).

In normal prostate samples, we found that the major transcription regulators were HOXB4, NFYB, and TFE3 (Supplementary Fig. 5a and Supplementary Fig. 5c), which differed from those in prostate cancer. In normal samples, the role of FOXA1, NFYA, and FOXP1 in regulating stemness genes was changed by comparing their results of PCA analysis in prostate cancer (Supplementary Fig. 5b and Fig. $3 c)$. These results indicated that the same transcription factors might play different roles in regulating the cell stemness in prostate normal and cancer samples.

Our transcriptional regulator analysis results showed that the upstream of the $E Z H 2$, the most relevant gene to the stemness of PCa cells, could be significantly bound by NFY (Fig. 3d). As a transcription factor, NFY regulates the self-renewal of hematopoietic stem cells [51] and promotes the self-renewal and expansion of prostate cancer cells and their stemness [50]. Hence EZH2 might play a stemness role in prostate cancer.

\section{Immunological microenvironment negatively related to the stemness of PCa cells}

By scoring the stemness and immunity of the immune microenvironment of PCa in different Gleason grades of clinical samples, there was a negative correlation between the PCa cell stemness and the immunity of the microenvironment of PCa clinical samples in all Gleason grade. The correlation coefficient of Gleason grade 1-2 was almost the same as that of Gleason grade 3-5 (Fig. 4a). By analyzing and scoring the stromal and immune cells of the PCa microenvironment, we found that the scores of stromal and immune cells in the PCa microenvironment were all inversely related to the stemness of PCa cells (Supplementary Fig. 6a). Although the immune score increased with the increase of Gleason grade, in each type of Gleason grade, the stemness of the low-immunity group was significantly increased than the stemness of the high-immunity group. (Supplementary Fig. 6b). After clustering the MEmangenta module genes based on cell stemness and immune scores of PCa, we found that PCa cell stemness was negatively related to the immunity of the PCa microenvironment in the clinical samples with high expression of genes of the MEmagenta module (Supplementary Fig. 6d). 
Furthermore, by analyzing and scoring the immune infiltration of PCa clinical samples from TCGA, the number of 22 types of immune cells in prostate cancer with different Gleason grades was obtained (Fig. 4b). The number of most types of immune cells in the microenvironment of PCa was increased with the Gleason grade increase; and CD8+ T cells [8] and macrophage M1 [9] were the most significantly increased in all types of immune cells, while the plasma cells [51] were reduced considerably with the Gleason grade increase (Fig. 4b), indicating that plasma cells (B cells) in the microenvironment of PCa played a vital role in anti-PCa immunity.

According to the results of immune cell correlation, immune cells were divided into 4 clusters by clustering. Fifteen immune cells were clustered in cluster-A, three immune cells were clustered in cluster-B, two immune cells were clustered in cluster- $C$, and two immune cells were clustered in cluster-D. And there were twelve edges in cluster-A, and the edges in other clusters were all connected to cluster-A. Therefore, cluster-A was more complicated in this immune cell network. For examples, the activated NK cell was negatively correlated with resting NK cell, which was most significant in all correlation among immune cells (Supplementary Fig. 6c), and the number of activated NK cell increased with the Gleason grade increase in $\mathrm{PCa}$ (Fig. 4b), indicating that activated NK cell might play a significant inhibitory function on prostate cancer stemness; the most significant positive correlation was between the activated dendritic cell and the memory B cell, and the number of both activated dendritic cells and memory B cells was all increased with the Gleason grade increase (Fig. 4c), which indicated that these two types of cells might play essential roles in inhibiting the stemness of PCa cells.

\section{The protein interaction network of stemness-related genes and its relationship with prostate cancer immune cells}

By screening and analyzing human protein interaction data containing MEmagenta module genes from the STRING database, we found the important protein-protein interaction network in the proteins of MEmagenta module genes and found that EZH2 interacted directly with 17 proteins. In the EZH2-related 17 protein-protein interactions, we also found that EZH2 could regulate the entire protein interaction network of MEmagenta module stemness genes by mainly interacting with CENPA, BUB1B, and PARP1 (Fig. 5a) [52-55]. Furthermore, function enrichment analysis of genes of the MEmagenta module revealed that most function of these genes was concentrated in cell mitosis, and the most significant functional pathway was related cell cycle (Supplementary Fig. 7a and Supplementary Fig. 7b), suggesting that these stemness genes might involve in the regulation of PCa stem cell mitosis.

From the analysis results of the relationship between the expression of the MEmagenta module gene and immune infiltration of PCa, we found that different immune cells had different effects on the expression of stemness genes in PCa. Different types of immune cells could affect the same stemness gene expression, and one stemness gene expression could also reversely affect different types of immune cells (Supplementary materials S5). In correlation of expression of stemness genes and immune cells, we found that expression of most stemness genes was positively correlated to memory $B$ cells and naive $B$ cells and negatively correlated to plasma cells (Fig. $5 b$ ). Further, the number of these B cells 
increased, while the number of plasma cells decreased, increasing the PCa Gleason grade (Fig. 4b). These results indicated that B cells might play the opposite effects on PCa cell stemness in different conditions (it's consistent with reference [56]). Besides, the number of activated NK cells and memory CD4+ T cells were all increased with the Gleason grade; and the expression of stemness genes of the MEmagenta module was positively correlated to resting NK cells and memory CD4+ T cells, while negatively correlated to activated NK cells (Fig. $4 b$ and $5 b$ ).

Expression of EZH2, the most relevant gene to PCa cell stemness, was most positively correlated to the activated memory CD4+ T cells and negatively correlated to the resting Mast cells (Fig. $5 \mathrm{c}$ and $5 \mathrm{~d}$ ). Most types of cells positively correlated to the expression of the EZH2 were T cells and B cells (Fig. $5 \mathrm{c}$ ), suggesting that $T$ cells and $B$ cells were the critical immune cells in regulating PCa cell stemness by controlling the expression of the EZH2. Further, the number of resting mast cells decreased, and the number of activated mast cells increased with the increased PCa Gleason grade (Fig. 4b); and the resting mast cells were the most negatively correlated to the expression of the EZH2 (Fig. $5 \mathrm{c}$ and $5 \mathrm{~d}$ ). These results indicated that the immunity and immune cells of the microenvironment of PCa played an essential role in the tumorigenesis and development of prostate cancer.

\section{Discussion}

It has been identified that cancer stem cells are closely related to cancer cell migration and play critical roles in tumor metastasis in PCa clinical samples [57]. The research on the stemness of PCa cells and their influencing factors is inevitably becoming a key tumor research direction. In this study, we found the gene module (MEmagenta module) most related to stemness of prostate cancer cells by analyzing multiomics of PCa clinical samples and cell lines, and also found that the CNVs and transcriptional regulators of the gene of the MEmagenta module were the critical influencing factors related to the stemness of prostate cancer cells by conjoint analysis of transcriptome data, gene CNV data and ATAC-seq data in prostate cancer. In the analysis results of the immune microenvironment of prostate cancer, it's found that the immune microenvironment of PCa played a key role and negatively correlated with the stemness of prostate cancer cells. The immune cells in the immune microenvironment of PCa had different correlations with the expression of stemness genes of the MEmagenta module. It's said that the immune microenvironment of PCa and its immune cells were also the essential influencing factors related to the stemness of PCa cells.

It's reported that tumor cell stemness can affect the immune response of the tumor immune microenvironment and then result in tumor heterogeneity [32]. Although there have been many studies on the relationship between tumor cell stemness and tumor development in other tumors, few studies on the relationship between tumor cell stemness and PCa development, especially on the factors affecting and regulating the cell stemness of prostate cancer. In this study, based on a variety of omics data, by fully using a variety of bioinformatics analysis software and methods, we not only obtained two factors related to PCa cell stemness, including the CNVs of stemness-related genes and the immunity of tumor microenvironment but also got the gene set most related to stemness of PCa cells. Many critical genes 
included in this gene set and their transcription regulators were important in regulating and affecting PCa metastasis by influencing PCa cell stemness. EZH2 promotes the formation of cancer stem cells and expands the aggressive cancer cell population, and leads to cancer progression [58].

Furthermore, EZH2 can also co-regulate prostate cancer stem cell properties with BRCA1 [59]. RUBVL1 is a gene related to the stemness of prostate cancer cells, and its copy number is increased with the tumor progresses. It is reported that RUVBL1 is essential for the survival of hematopoietic stem cells [40], and its gene copy number is increased in head and neck squamous cancers [60]. MYBL2 is helpful in DNA double-strand repair in hematopoietic stem cells [61] and the emergence of CNV leds to the occurrence and development of cancer $[62,63]$. AURKB determines the identity of embryonic stem cells [64], and the deletion of the copy number also contributes to the formation of aggressive tumors [65]. PUM1 regulates the expression of hematopoietic stem cells [66] and promotes the migration of cancer cells [47]. CLOCK regulates the biological clock of cancer stem cells and promotes the self-renewal of cancer cells [67]. HMGCS1 promotes cancer development [68] and affects the function of NK cells [69]. SUV39H1 attenuates the apoptosis of cancer cells [70] and enhances the immune escape of tumor cells [71]. In addition to the reported genes related to cancer cell stemness, our results also showed many genes that had not been reported to be related to cancer cell stemness, especially to PCa cell stemness, such as TEDC2, TMEM132A, and VARS, etc.

In prostate cancer, up to now, there are few studies on the relationship between cell stemness regulatory factors and tumor malignancy. From our multi-omics analysis results, the CNVs of cancer cell stemness-related genes were near related to the malignant degree of prostate cancer (Gleason grade). The CNVs of genes positively related to PCa cell stemness were also positively correlated with the degree of malignant prostate cancer (Gleason grade); otherwise, the CNVs of genes negatively associated with PCa cell stemness was negatively correlated with Gleason grades of prostate cancers. The correlations between transcriptional regulators of PCa cell stemness genes and prostate tumor malignancy were similar to the correlation between CNVs of PCa cell stemness genes and prostate tumor malignancy.

Although we found that the CNVs and transcriptional regulations of PCa cell stemness genes and the immune infiltration of prostate cancer were all important factors in influencing the stemness of PCa cells and further regulating the development of PCa and we also analyzed and obtained the key gene set and genes in regulating the stemness of PCa cells, the experimental evidence and the detailed mechanism of the correlation and regulation among CNVs of PCa cell stemness genes, transcription regulators of PCa cell stemness genes and immunity of PCa microenvironment were still unclear and needed to be further investigated in the future.

\section{Conclusions}

Our research found that samples with high Gleason grade of prostate cancer had high CNV and high stemness score, and low immune microenvironment was beneficial to the stemness of prostate cancer cells. Analysis results also demonstrated that some transcription factors of the most important stemness 
genes enhanced the regulation of stemness of prostate cancer cells. However, its detailed molecular mechanism needs future experimental verification in vivo and in vitro.

\section{List Of Abbreviations}

pCSC: Prostate cancer stem cells; PCa: Prostate cancer; WGCNA: Weighted Gene Co-expression Network Analysis; STRING: functional protein association networks; CNVs: Copy Number Variants; CSCs: Cancer stem cells; PPI: Protein-Protein Interaction; SCPC: small cell prostate cancer; PRAD: prostate adenocarcinoma;

\section{Declarations}

\section{Ethics approval and consent to participate}

Not applicable

\section{Acknowledgments}

Thanks to Dr. Wei Guanyun for her suggestions on data analysis.

\section{Funding}

This research was funded by the National Natural Science Foundation of China (NNSFC) (grant no. 81872104 and 81472415$)$.

\section{Authors' contributions}

Data analysis, writing original draft Zao Dai; Review and revised the manuscript, Ping Liu.

\section{Availability of data and materials}

All data generated or analyzed during this study are available.

\section{Consent for publication}

Not applicable

\section{Competing interests}

The authors declare no conflict of interest.

\section{Data statement}

All data generated or analyzed during this study are included in this article. 


\section{References}

1. Friedmann-Morvinski D, Verma IM: Dedifferentiation and reprogramming: origins of cancer stem cells. EMBO reports 2014, 15:244-253.

2. Malta TM, Sokolov A, Gentles AJ, Burzykowski T, Poisson L, Weinstein JN, Kaminska B, Huelsken J, Omberg L, Gevaert O, et al: Machine Learning Identifies Stemness Features Associated with Oncogenic Dedifferentiation. Cell 2018, 173:338-354 e315.

3. Skvortsov S, Skvortsova I-I, Tang DG, Dubrovska A: Concise Review: Prostate Cancer Stem Cells: Current Understanding. Stem Cells 2018, 36:1457-1474.

4. Rycaj K, Tang DG: Cell-of-Origin of Cancer versus Cancer Stem Cells: Assays and Interpretations. Cancer Res 2015, 75:4003.

5. Mei W, Lin X, Kapoor A, Gu Y, Zhao K, Tang D: The Contributions of Prostate Cancer Stem Cells in Prostate Cancer Initiation and Metastasis. Cancers (Basel) 2019, 11.

6. Liao C-P, Adisetiyo H, Liang M, Roy-Burman P: Cancer Stem Cells and Microenvironment in Prostate Cancer Progression. Hormones and Cancer 2010, 1:297-305.

7. Krueger TE, Thorek DLJ, Meeker AK, Isaacs JT, Brennen WN: Tumor-infiltrating mesenchymal stem cells: Drivers of the immunosuppressive tumor microenvironment in prostate cancer? The Prostate 2019, 79:320-330.

8. Wang W, Green M, Choi JE, Gijón M, Kennedy PD, Johnson JK, Liao P, Lang X, Kryczek I, Sell A, et al: CD8+ T cells regulate tumour ferroptosis during cancer immunotherapy. Nature 2019, 569:270-274.

9. Mills CD, Lenz LL, Harris RA: A Breakthrough: Macrophage-Directed Cancer Immunotherapy. Cancer Res 2016, 76:513.

10. Ihle $\mathrm{CL}$, Owens $\mathrm{P}$ : Integrating the immune microenvironment of prostate cancer induced bone disease. Mol Carcinog 2020, 59:822-829.

11. Shiao SL, Chu GC, Chung LW: Regulation of prostate cancer progression by the tumor microenvironment. Cancer Lett 2016, 380:340-348.

12. Weng CC, Ding PY, Liu YH, Hawse JR, Subramaniam M, Wu CC, Lin YC, Chen CY, Hung WC, Cheng KH: Mutant Kras-induced upregulation of CD24 enhances prostate cancer stemness and bone metastasis. Oncogene 2019, 38:2005-2019.

13. Pai VC, Hsu CC, Chan TS, Liao WY, Chuu CP, Chen WY, Li CR, Lin CY, Huang SP, Chen LT, Tsai KK: ASPM promotes prostate cancer stemness and progression by augmenting Wnt-Dvl-3-beta-catenin signaling. Oncogene 2019, 38:1340-1353.

14. Xu N, Wu YP, Yin HB, Xue XY, Gou X: Molecular network-based identification of competing endogenous RNAs and mRNA signatures that predict survival in prostate cancer. J Trans/ Med 2018, 16:274.

15. Zhu X, Gou X, Zhou M: Nomograms Predict Survival Advantages of Gleason Score 3+4 Over 4+3 for Prostate Cancer: A SEER-Based Study. Front Oncol 2019, 9:646. 
16. Cancer Genome Atlas Research N: The Molecular Taxonomy of Primary Prostate Cancer. Cel/2015, 163:1011-1025.

17. He Z, Tang F, Lu Z, Huang Y, Lei H, Li Z, Zeng G: Analysis of differentially expressed genes, clinical value and biological pathways in prostate cancer. Am J Trans/ Res 2018, 10:1444-1456.

18. He Z, Duan X, Zeng G: Identification of potential biomarkers and pivotal biological pathways for prostate cancer using bioinformatics analysis methods. PeerJ 2019, 7:e7872.

19. Langfelder P, Horvath S: WGCNA: an R package for weighted correlation network analysis. $B M C$ Bioinformatics 2008, 9:559.

20. Kim D, Paggi JM, Park C, Bennett C, Salzberg SL: Graph-based genome alignment and genotyping with HISAT2 and HISAT-genotype. Nat Biotechnol 2019, 37:907-915.

21. Anders S, Pyl PT, Huber W: HTSeq-a Python framework to work with high-throughput sequencing data. Bioinformatics 2015, 31:166-169.

22. Mermel CH, Schumacher SE, Hill B, Meyerson ML, Beroukhim R, Getz G: GISTIC2.0 facilitates sensitive and confident localization of the targets of focal somatic copy-number alteration in human cancers. Genome Biology 2011, 12:R41.

23. Langmead B, Salzberg SL: Fast gapped-read alignment with Bowtie 2. Nat Methods 2012, 9:357-359.

24. Ramírez F, Dündar F, Diehl S, Grüning BA, Manke T: deepTools: a flexible platform for exploring deepsequencing data. Nucleic Acids Res 2014, 42:W187-W191.

25. Zhang Y, Liu T, Meyer CA, Eeckhoute J, Johnson DS, Bernstein BE, Nusbaum C, Myers RM, Brown M, Li W, Liu XS: Model-based Analysis of ChIP-Seq (MACS). Genome Biology 2008, 9:R137.

26. Heinz S, Benner C, Spann N, Bertolino E, Lin YC, Laslo P, Cheng JX, Murre C, Singh H, Glass CK: Simple combinations of lineage-determining transcription factors prime cis-regulatory elements required for macrophage and B cell identities. Mol Cell 2010, 38:576-589.

27. Phanstiel DH, Boyle AP, Araya CL, Snyder MP: Sushi.R: flexible, quantitative and integrative genomic visualizations for publication-quality multi-panel figures. Bioinformatics 2014, 30:2808-2810.

28. Yoshihara K, Shahmoradgoli M, Martínez E, Vegesna R, Kim H, Torres-Garcia W, Treviño V, Shen H, Laird PW, Levine DA, et al: Inferring tumour purity and stromal and immune cell admixture from expression data. Nature Communications 2013, 4:2612.

29. Newman AM, Liu CL, Green MR, Gentles AJ, Feng W, Xu Y, Hoang CD, Diehn M, Alizadeh AA: Robust enumeration of cell subsets from tissue expression profiles. Nat Methods 2015, 12:453-457.

30. Shannon P, Markiel A, Ozier O, Baliga NS, Wang JT, Ramage D, Amin N, Schwikowski B, Ideker T: Cytoscape: A Software Environment for Integrated Models of Biomolecular Interaction Networks. Genome Res 2003, 13:2498-2504.

31. Yu G, Wang LG, Han Y, He QY: clusterProfiler: an R package for comparing biological themes among gene clusters. OMICS 2012, 16:284-287.

32. Miranda A, Hamilton PT, Zhang AW, Pattnaik S, Becht E, Mezheyeuski A, Bruun J, Micke P, de Reynies A, Nelson BH: Cancer stemness, intratumoral heterogeneity, and immune response across cancers. 
Proceedings of the National Academy of Sciences 2019, 116:9020.

33. Gorodetska I, Lukiyanchuk V, Peitzsch C, Kozeretska I, Dubrovska A: BRCA1 and EZH2 cooperate in regulation of prostate cancer stem cell phenotype. Int J Cancer 2019, 145:2974-2985.

34. Song I-S, Jeong YJ, Jeong SH, Heo HJ, Kim HK, Bae KB, Park Y-H, Kim SU, Kim J-M, Kim N, et al: FOXM1-Induced PRX3 Regulates Stemness and Survival of Colon Cancer Cells via Maintenance of Mitochondrial Function. Gastroenterology 2015, 149:1006-1016.e1009.

35. Zhang Q, Huang H, Liu A, Li J, Liu C, Sun B, Chen L, Gao Y, Xu D, Su C: Cell division cycle 20 (CDC20) drives prostate cancer progression via stabilization of $\beta$-catenin in cancer stem-like cells. EBioMedicine 2019, 42:397-407.

36. Dai C, Miao CX, Xu XM, Liu LJ, Gu YF, Zhou D, Chen LS, Lin G, Lu GX: Transcriptional activation of human CDCA8 gene regulated by transcription factor NF-Y in embryonic stem cells and cancer cells. J Biol Chem 2015, 290:22423-22434.

37. Naef V, Monticelli S, Corsinovi D, Mazzetto MT, Cellerino A, Ori M: The age-regulated zinc finger factor ZNF367 is a new modulator of neuroblast proliferation during embryonic neurogenesis. Sci Rep 2018, 8:11836.

38. Hu R, Wang MQ, Niu WB, Wang YJ, Liu YY, Liu LY, Wang M, Zhong J, You HY, Wu XH, et al: SKA3 promotes cell proliferation and migration in cervical cancer by activating the PI3K/Akt signaling pathway. Cancer Cell Int 2018, 18:183.

39. Taniuchi K, Furihata M, Iwasaki S, Tanaka K, Shimizu T, Saito M, Saibara T: RUVBL1 directly binds actin filaments and induces formation of cell protrusions to promote pancreatic cancer cell invasion. Int J Oncol 2014, 44:1945-1954.

40. Bereshchenko O, Mancini E, Luciani L, Gambardella A, Riccardi C, Nerlov C: Pontin is essential for murine hematopoietic stem cell survival. Haematologica 2012, 97:1291-1294.

41. Liu M, Hu Q, Tu M, Wang X, Yang Z, Yang G, Luo R: MCM6 promotes metastasis of hepatocellular carcinoma via MEK/ERK pathway and serves as a novel serum biomarker for early recurrence. $J$ Exp Clin Cancer Res 2018, 37:10.

42. Barton KM, Levine EM: Expression patterns and cell cycle profiles of PCNA, MCM6, cyclin D1, cyclin A2, cyclin B1, and phosphorylated histone H3 in the developing mouse retina. Dev Dyn 2008, 237:672-682.

43. Tomonaga T, Matsushita K, Ishibashi M, Nezu M, Shimada H, Ochiai T, Yoda K, Nomura F: Centromere protein $\mathrm{H}$ is up-regulated in primary human colorectal cancer and its overexpression induces aneuploidy. Cancer Res 2005, 65:4683-4689.

44. Zhang JP, Zhang H, Wang HB, Li YX, Liu GH, Xing S, Li MZ, Zeng MS: Down-regulation of Sp1 suppresses cell proliferation, clonogenicity and the expressions of stem cell markers in nasopharyngeal carcinoma. J Transl Med 2014, 12:222.

45. Zhao WF, Wang HB, Xie B, Hu LJ, Xu LH, Kuang BH, Li MZ, Zhang X: Sp1 and Sp3 are involved in the full transcriptional activity of centromere protein $\mathrm{H}$ in human nasopharyngeal carcinoma cells. FEBS J 2012, 279:2714-2726. 
46. Uyhazi KE, Yang Y, Liu N, Qi H, Huang XA, Mak W, Weatherbee SD, Song X, Lin H: Pumilio Proteins Exert Distinct Biological Functions and Multiple Modes of Post-Transcriptional Regulation in Embryonic Stem Cell Pluripotency and Early Embryogenesis. bioRxiv 2019:751909.

47. Guan X, Chen S, Liu Y, Wang L-I, Zhao Y, Zong Z-H: PUM1 promotes ovarian cancer proliferation, migration and invasion. Biochem Biophys Res Commun 2018, 497:313-318.

48. Janich P, Pascual G, Merlos-Suárez A, Batlle E, Ripperger J, Albrecht U, Cheng H-YM, Obrietan K, Di Croce L, Benitah SA: The circadian molecular clock creates epidermal stem cell heterogeneity. Nature 2011, 480:209-214.

49. Gambara G, Desideri M, Stoppacciaro A, Padula F, De Cesaris P, Starace D, Tubaro A, del Bufalo D, Filippini A, Ziparo E, Riccioli A: TLR3 engagement induces IRF-3-dependent apoptosis in androgensensitive prostate cancer cells and inhibits tumour growth in vivo. J Cell Mol Med 2015, 19:327-339.

50. Blum R, Gupta R, Burger PE, Ontiveros CS, Salm SN, Xiong X, Kamb A, Wesche H, Marshall L, Cutler G, et al: Molecular Signatures of Prostate Stem Cells Reveal Novel Signaling Pathways and Provide Insights into Prostate Cancer. PLOS ONE 2009, 4:e5722.

51. Wouters MCA, Nelson BH: Prognostic Significance of Tumor-Infiltrating B Cells and Plasma Cells in Human Cancer. Clin Cancer Res 2018, 24:6125.

52. García del Arco A, Edgar BA, Erhardt S: In Vivo Analysis of Centromeric Proteins Reveals a Stem CellSpecific Asymmetry and an Essential Role in Differentiated, Non-proliferating Cells. Cell Reports 2018, 22:1982-1993.

53. Behnan J, Grieg Z, Joel M, Ramsness I, Stangeland B: Gene knockdown of CENPA reduces sphere forming ability and stemness of glioblastoma initiating cells. Neuroepigenetics 2016, 7:6-18.

54. Venere M, Miller TE, Rich JN: Mitotic Control of Cancer Stem Cells. Cancer Discovery 2013, 3:141.

55. Hsieh M-H, Chen Y-T, Chen Y-T, Lee Y-H, Lu J, Chien C-L, Chen H-F, Ho H-N, Yu C-J, Wang Z-Q, Teng SC: PARP1 controls KLF4-mediated telomerase expression in stem cells and cancer cells. Nucleic Acids Res 2017, 45:10492-10503.

56. Sharonov GV, Serebrovskaya EO, Yuzhakova DV, Britanova OV, Chudakov DM: B cells, plasma cells and antibody repertoires in the tumour microenvironment. Nature Reviews Immunology 2020, 20:294-307.

57. Horning AM, Wang Y, Lin CK, Louie AD, Jadhav RR, Hung CN, Wang CM, Lin CL, Kirma NB, Liss MA, et al: Single-Cell RNA-seq Reveals a Subpopulation of Prostate Cancer Cells with Enhanced Cell-CycleRelated Transcription and Attenuated Androgen Response. Cancer Res 2018, 78:853-864.

58. Chang CJ, Hung MC: The role of EZH2 in tumour progression. Br J Cancer 2012, 106:243-247.

59. Gorodetska I, Lukiyanchuk V, Peitzsch C, Kozeretska I, Dubrovska A: BRCA1 and EZH2 cooperate in regulation of prostate cancer stem cell phenotype. Int J Cancer 2019, 145:2974-2985.

60. Lin D, Lin B, Bhanot H, Riou R, Abt NB, Rajagopal J, Saladi SV: RUVBL1 is an amplified epigenetic factor promoting proliferation and inhibiting differentiation program in head and neck squamous cancers. Oral Oncol 2020, 111:104930. 
61. Bayley R, Blakemore D, Cancian L, Dumon S, Volpe G, Ward C, Almaghrabi R, Gujar J, Reeve N, Raghavan M, et al: MYBL2 Supports DNA Double Strand Break Repair in Hematopoietic Stem Cells. Cancer Res 2018, 78:5767-5779.

62. Musa J, Aynaud MM, Mirabeau O, Delattre O, Grunewald TG: MYBL2 (B-Myb): a central regulator of cell proliferation, cell survival and differentiation involved in tumorigenesis. Cell Death Dis 2017, 8:e2895.

63. Nakajima T, Yasui K, Zen K, Inagaki Y, Fujii H, Minami M, Tanaka S, Taniwaki M, Itoh Y, Arii S, et al: Activation of B-Myb by E2F1 in hepatocellular carcinoma. Hepatol Res 2008, 38:886-895.

64. Shin J, Kim TW, Kim H, Kim HJ, Suh MY, Lee S, Lee HT, Kwak S, Lee SE, Lee JH, et al: Aurkb/PP1mediated resetting of Oct4 during the cell cycle determines the identity of embryonic stem cells. Elife 2016, 5:e10877.

65. Hegyi K, Egervari K, Sandor Z, Mehes G: Aurora kinase B expression in breast carcinoma: cell kinetic and genetic aspects. Pathobiology 2012, 79:314-322.

66. Naudin C, Hattabi A, Michelet F, Miri-Nezhad A, Benyoucef A, Pflumio F, Guillonneau F, Fichelson S, Vigon I, Dusanter-Fourt I, Lauret E: PUMILIO/FOXP1 signaling drives expansion of hematopoietic stem/progenitor and leukemia cells. Blood 2017, 129:2493-2506.

67. Dong Z, Zhang G, Qu M, Gimple RC, Wu Q, Qiu Z, Prager BC, Wang X, Kim LJY, Morton AR, et al: Targeting Glioblastoma Stem Cells through Disruption of the Circadian Clock. Cancer Discov 2019, 9:1556-1573.

68. Ashida S, Kawada C, Inoue K: Stromal regulation of prostate cancer cell growth by mevalonate pathway enzymes HMGCS1 and HMGCR. Oncol Lett 2017, 14:6533-6542.

69. Assmann N, O'Brien KL, Donnelly RP, Dyck L, Zaiatz-Bittencourt V, Loftus RM, Heinrich P, Oefner PJ, Lynch L, Gardiner CM, et al: Srebp-controlled glucose metabolism is essential for NK cell functional responses. Nat Immunol 2017, 18:1197-1206.

70. Watson GW, Wickramasekara S, Palomera-Sanchez Z, Black C, Maier CS, Williams DE, Dashwood RH, Ho E: SUV39H1/H3K9me3 attenuates sulforaphane-induced apoptotic signaling in PC3 prostate cancer cells. Oncogenesis 2014, 3:e131.

71. Lu C, Yang D, Klement JD, Oh IK, Savage NM, Waller JL, Colby AH, Grinstaff MW, Oberlies NH, Pearce CJ, et al: SUV39H1 Represses the Expression of Cytotoxic T-Lymphocyte Effector Genes to Promote Colon Tumor Immune Evasion. Cancer Immunol Res 2019, 7:414-427.

\section{Figures}


$\mathrm{a}$

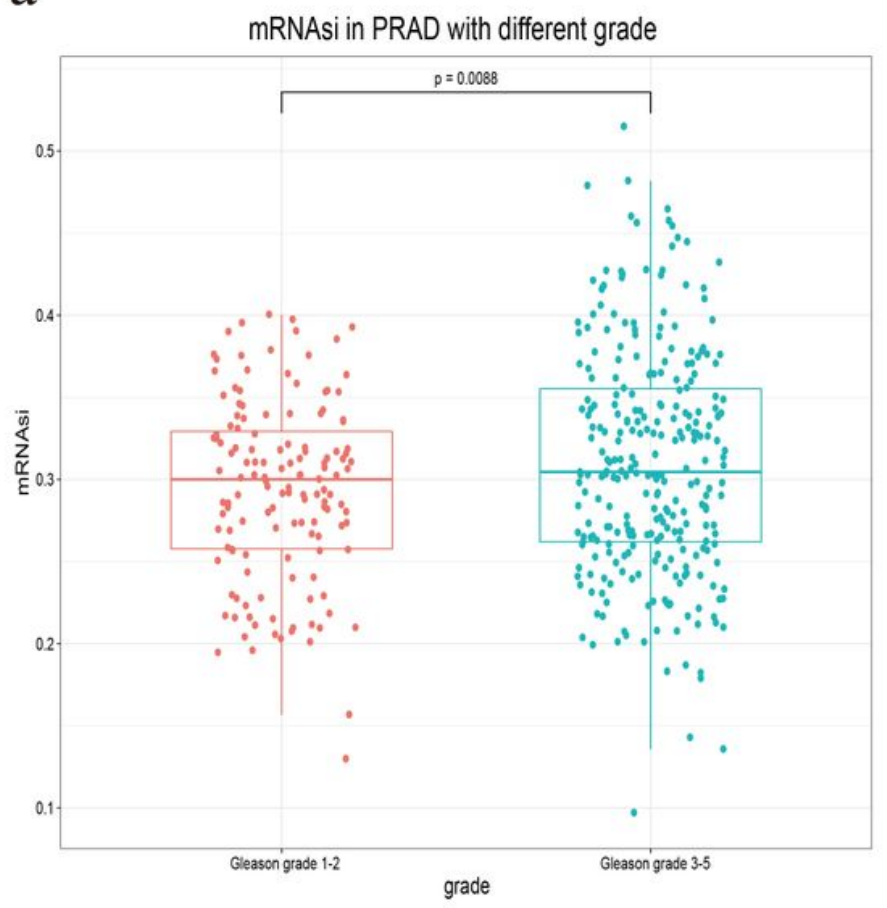

b

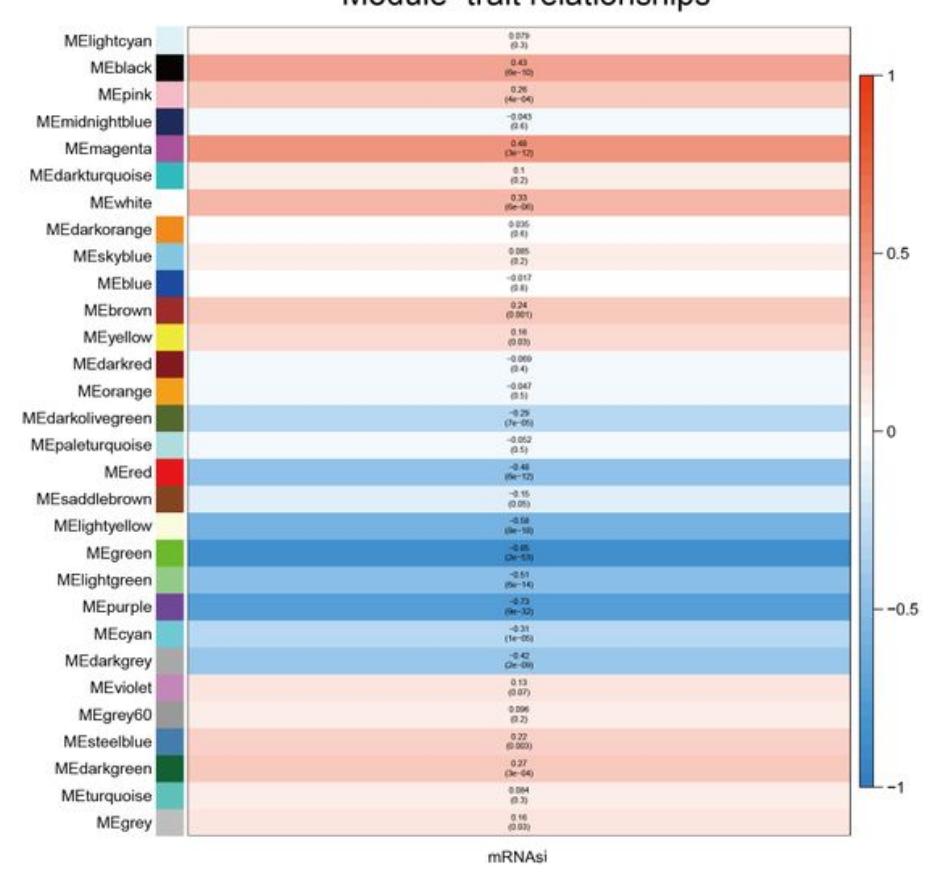

C

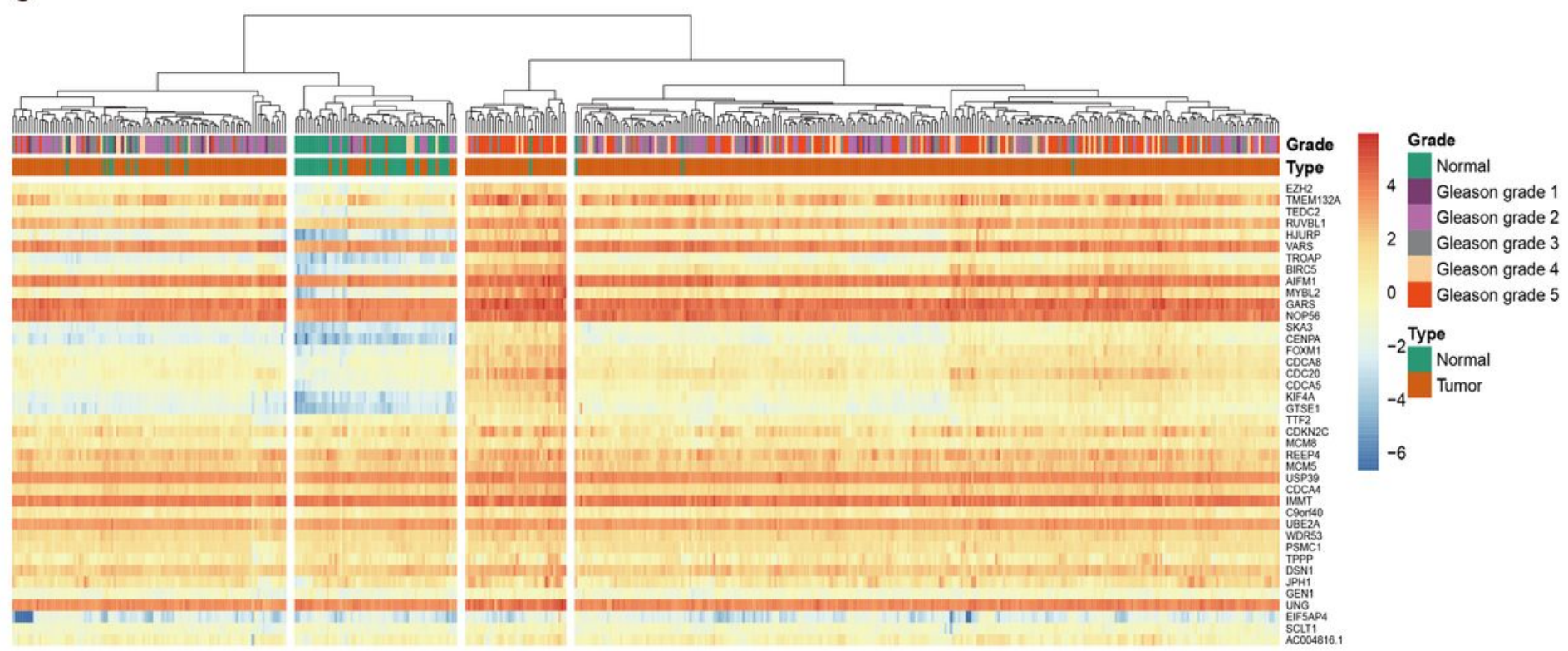

\section{Figure 1}

The relationship between stemness score and module and Gleason grade a The relationship between prostate cancer stemness score and Gleason grade. b The gene module related to stemness is based on the WGCNA method. Red represents a positive correlation between gene module and stemness, and blue represents a negative correlation between gene module and stemness. c The heatmap of genes in MEmagenta module, red represents high gene expression, and blue represents low gene expression 
a

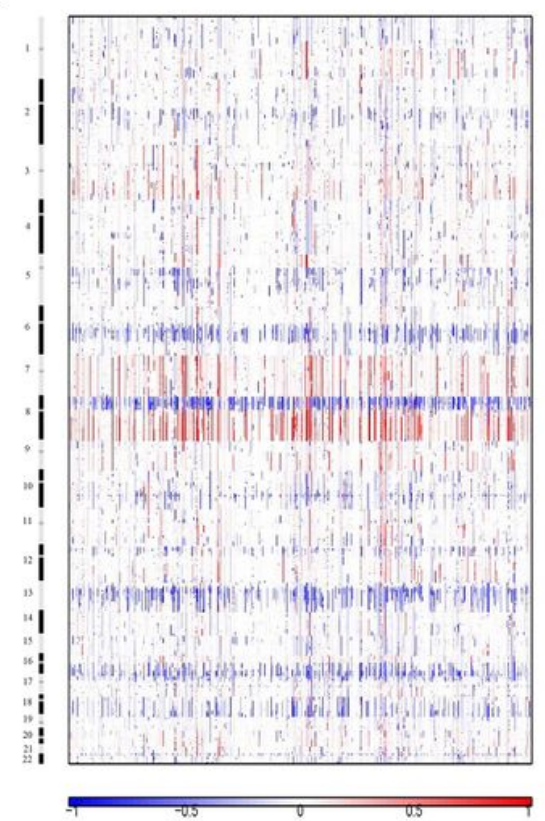

$\mathrm{b}$

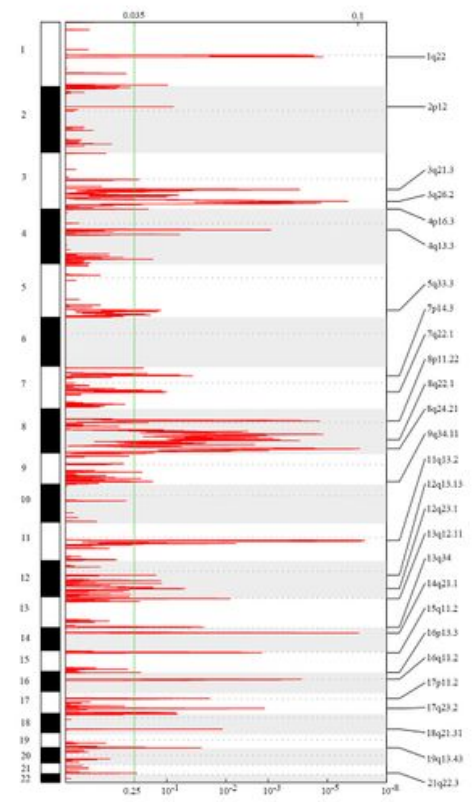

c

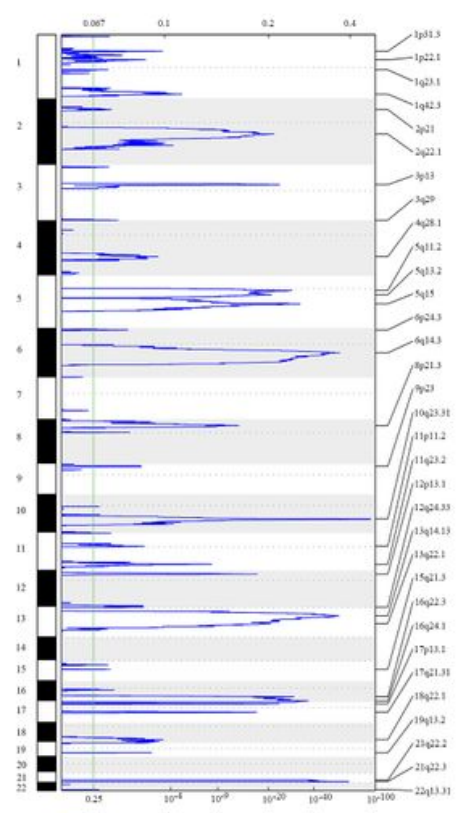

d

PRAD Gleason grade $1, n=23$

PRAD Gleason grade 2, $\mathrm{n}=116$
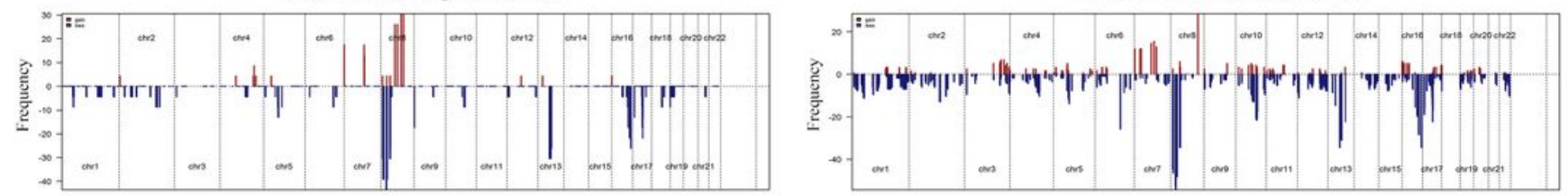

PRAD Gleason grade $3, \mathrm{n}=88$

PRAD Gleason grade $4, n=58$
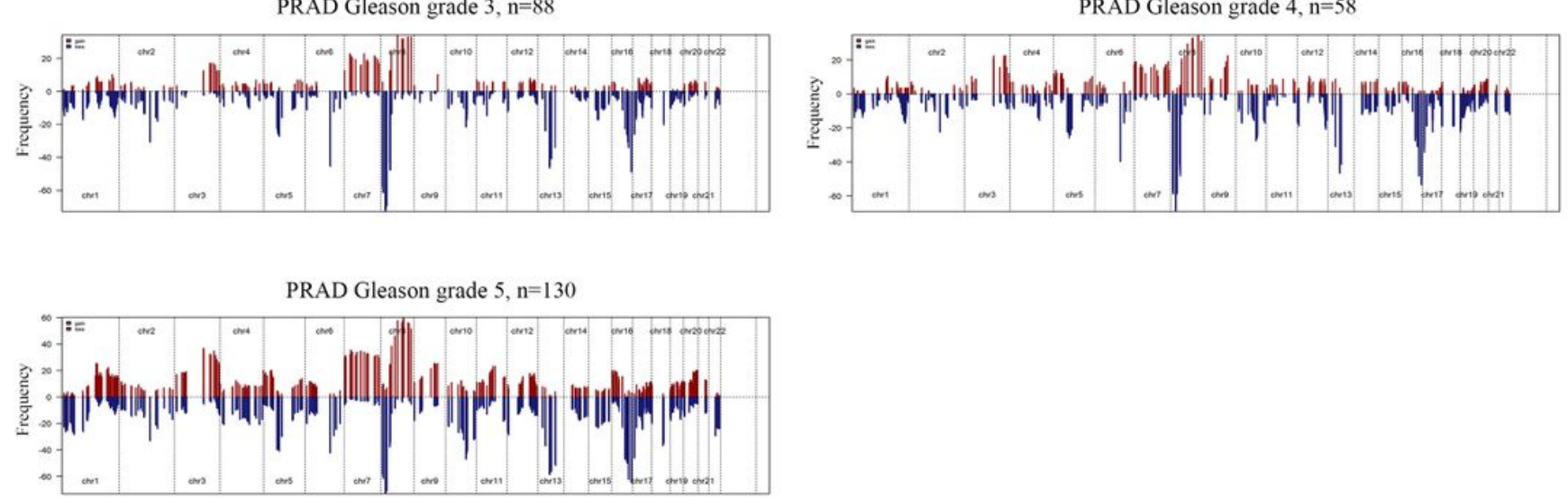

\section{Figure 2}

The stem gene CNV is related to cell stemness and malignancy of prostate cancer $a, b$, and c Changes of stem gene CNV in tumor samples. Red and blue represent the two types of CNV, amplification and deletion, respectively. $d$ The stem gene CNV increases with the increase of clinical grade. Red and blue represent the two types of CNV, amplification and deletion, respectively. 
a
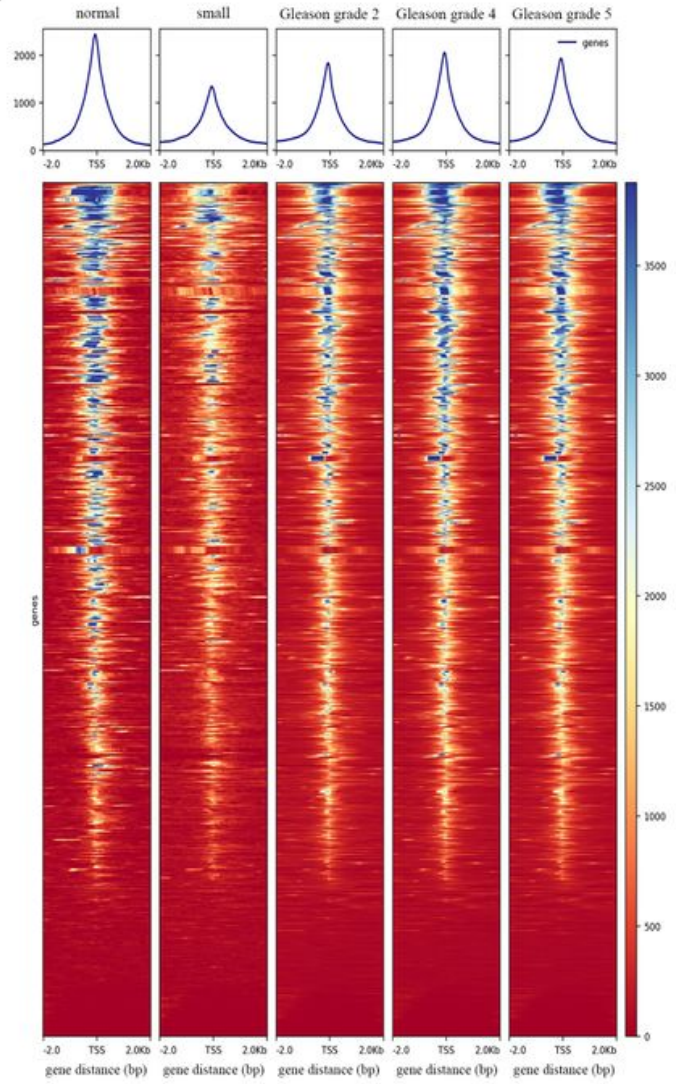

b

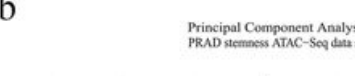

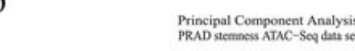

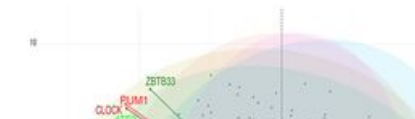

d

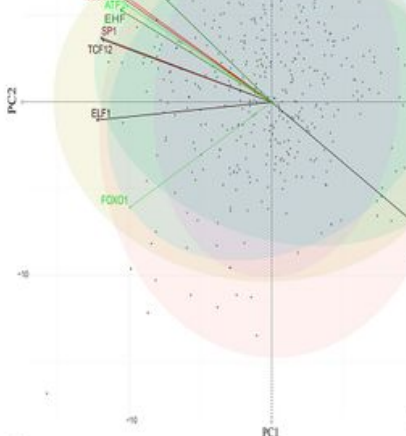
chr7

148,885

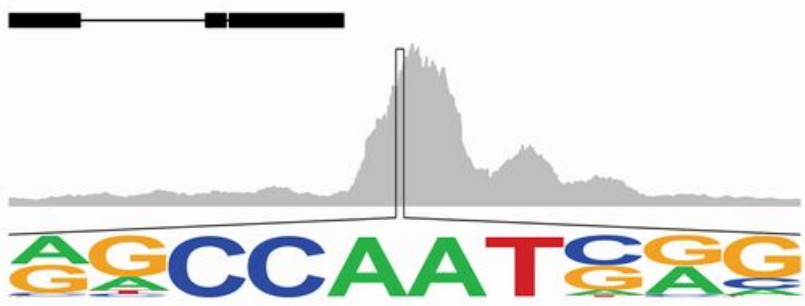

\section{Figure 3}

Transcriptional regulation of stemness gene in prostate cancer a In normal samples, cancer samples of different Gleason grades, and prostate small cell cancer samples, the transcription factor binding intensity of stemness gene. b The main transcriptional regulators are obtained based on PCA analysis. Red and green represent the strength and weakness of transcriptional regulatory factors, respectively. C Based on PCA analysis, the importance of known transcriptional regulators of stemness genes in prostate cancer. The shades of blue represent the correlation between transcription factors and stemness. D Motif map of transcription factor binding to EZH2. 

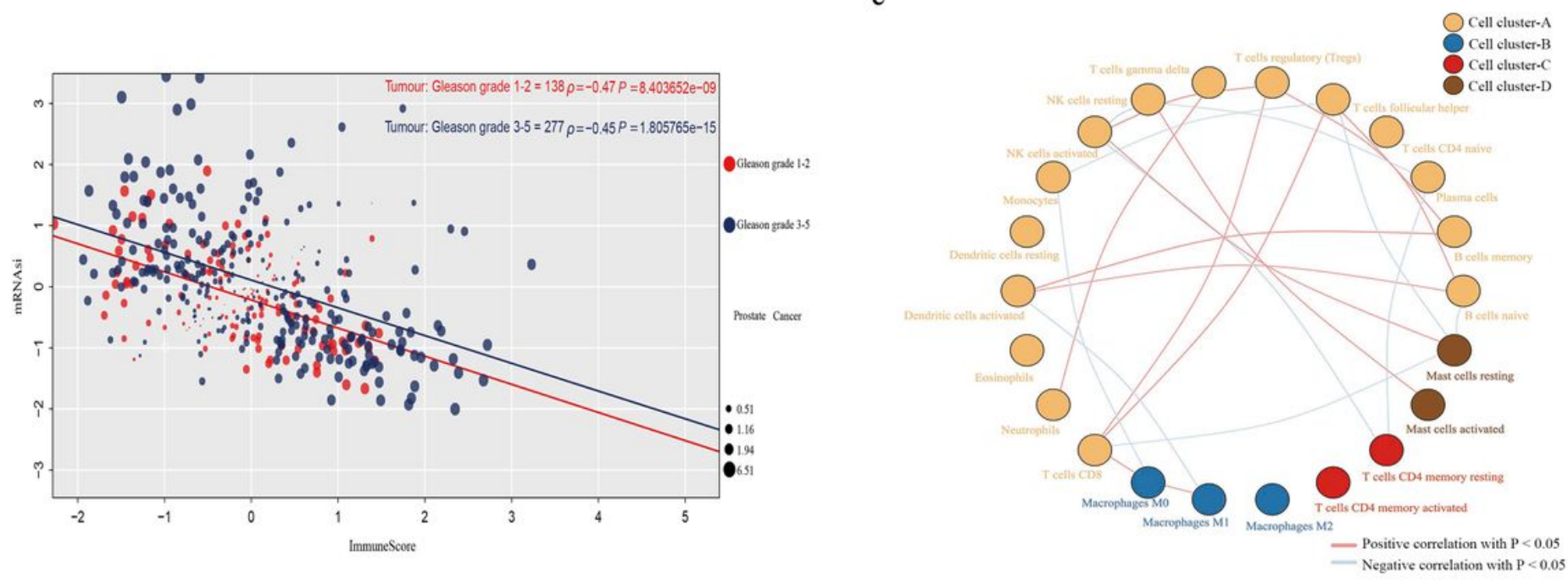

b

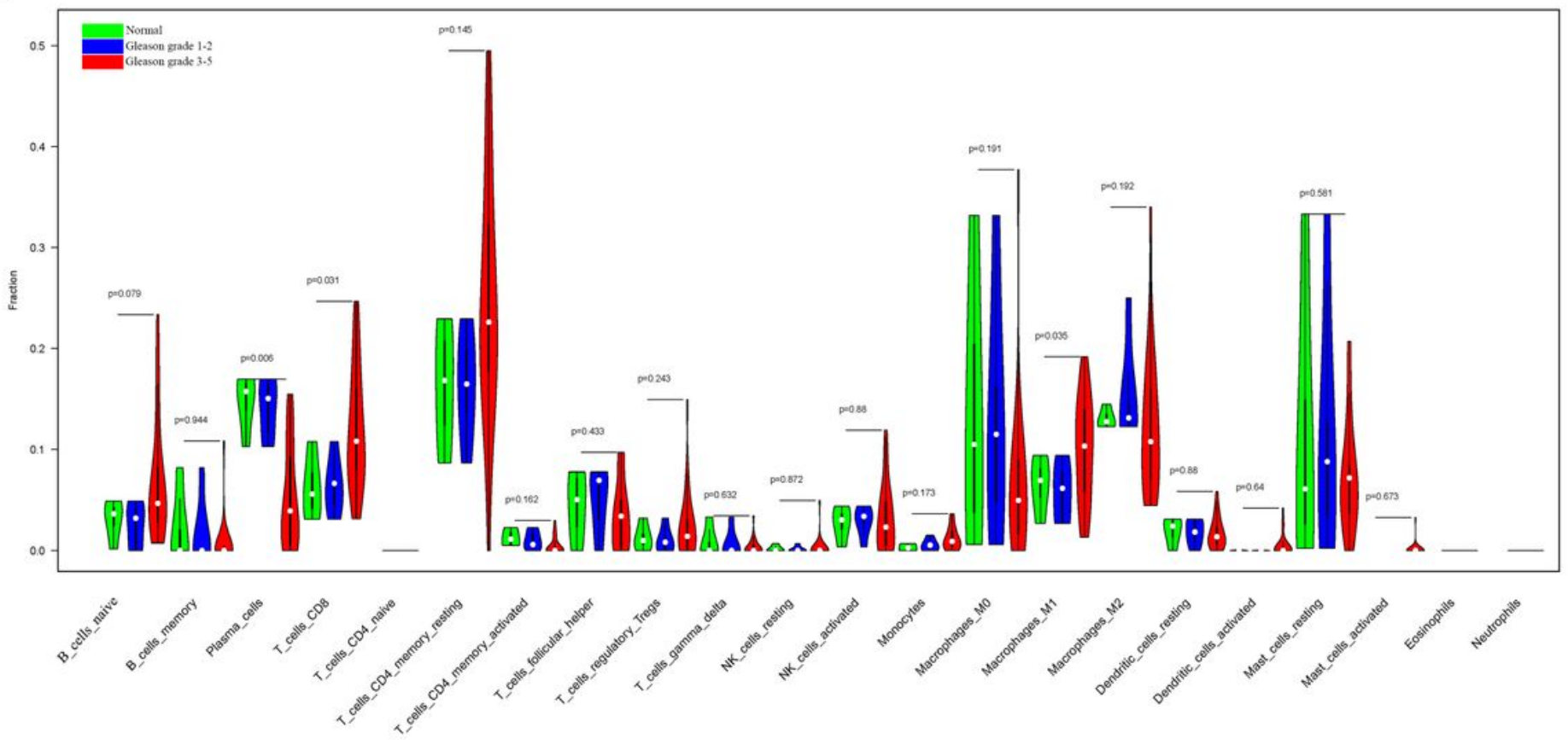

Figure 4

Stemness and immune cells in prostate cancer a In different Gleason grades of prostate cancer, stemness score is negatively correlated with immune infiltration score. $b$ In the prostate tumor microenvironment of different Gleason grades, the fraction of 22 types of immune cells. c Correlation network of 22 types of immune cells. 
a

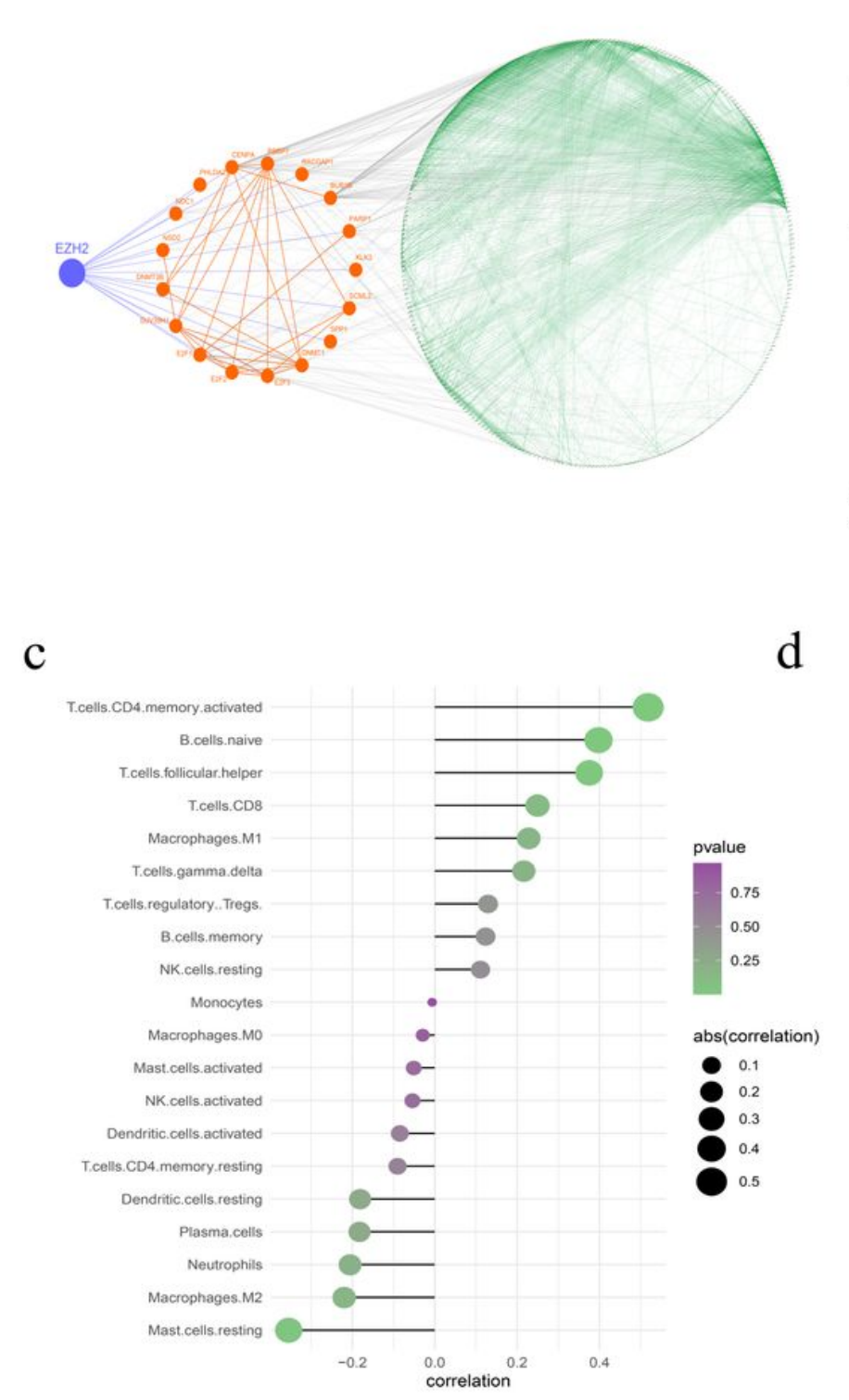

b

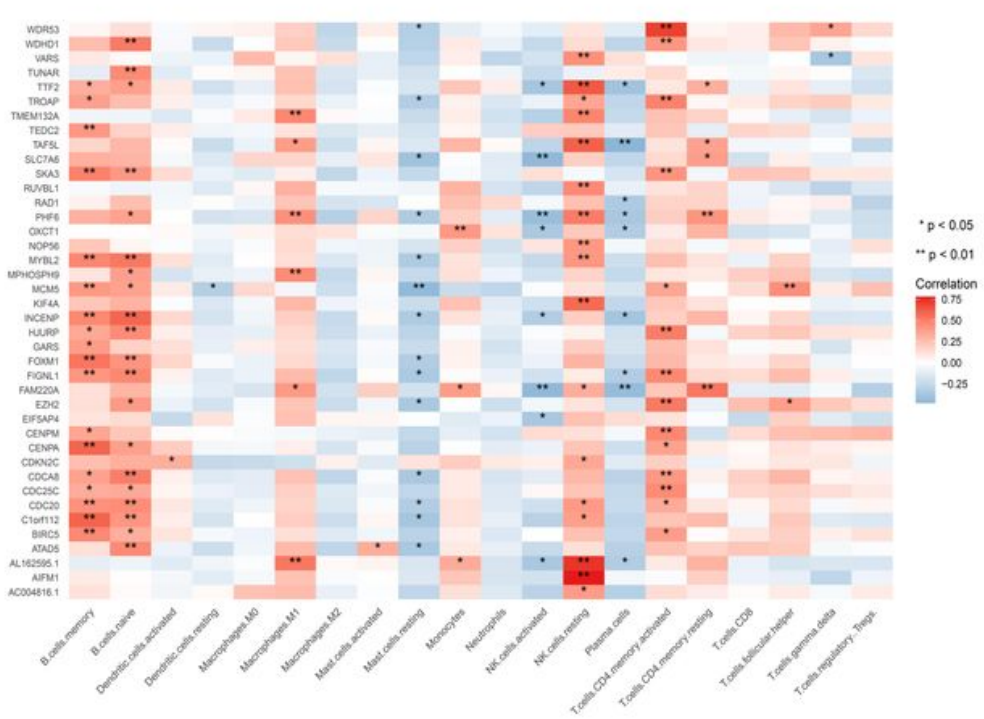

$\mathrm{n}=40, \mathrm{r}=-0.35$, p.value $=0.02$

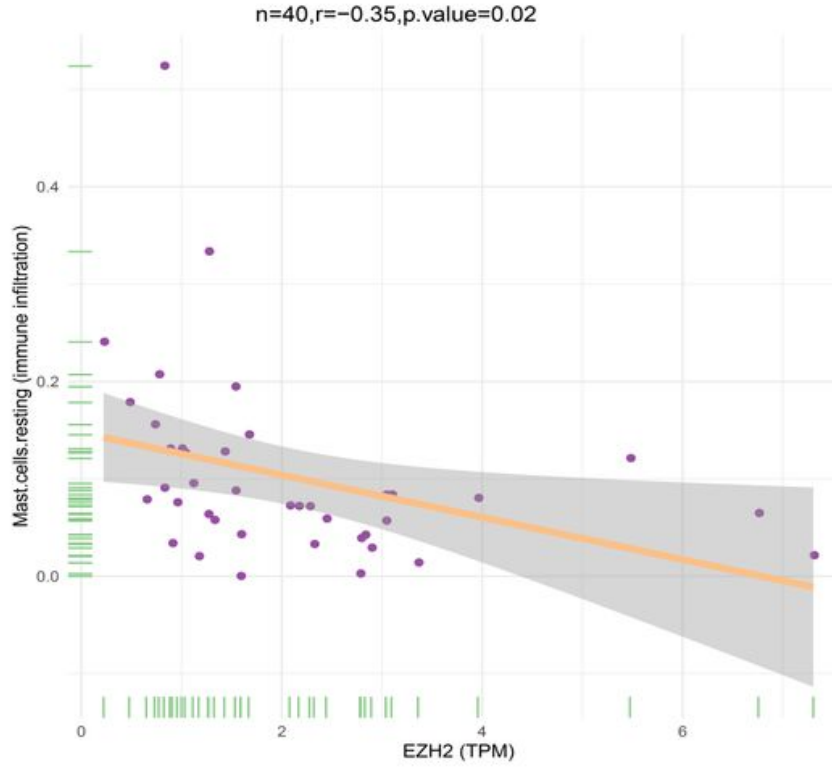

\section{Figure 5}

The protein interaction network of stemness gene, the relationship between stemness gene and immune infiltration a The connection between stemness genes and EZH2 in MEmagenta module. According to whether genes interact directly with EZH2, it is divided into a small circle and a large circle. The blue lines represent genes that directly interact with $\mathrm{EZH} 2$. The yellow lines represent the interaction between genes in the small circle, and the green lines represent the interaction between genes in the large circle. The gray lines represent the interaction of genes between the small circle and the large circle. b The correlation between stemness gene and immune cells, red represents positive correlation, blue represents negative correlation $\mathrm{c}$ and $\mathrm{d}$ The correlation between EZH2 and immune cells, and the correlation between EZH2 and resting mast cell

\section{Supplementary Files}


This is a list of supplementary files associated with this preprint. Click to download.

- SupplementaryFile.xlsx

- SupplementFigure1.jpg

- SupplementFigure2.jpg

- SupplementaryFigure3.jpg

- SupplementaryFigure4.jpg

- SupplementaryFigure5.jpg

- SupplementaryFigure6.jpg

- SupplementaryFigure7.jpg 